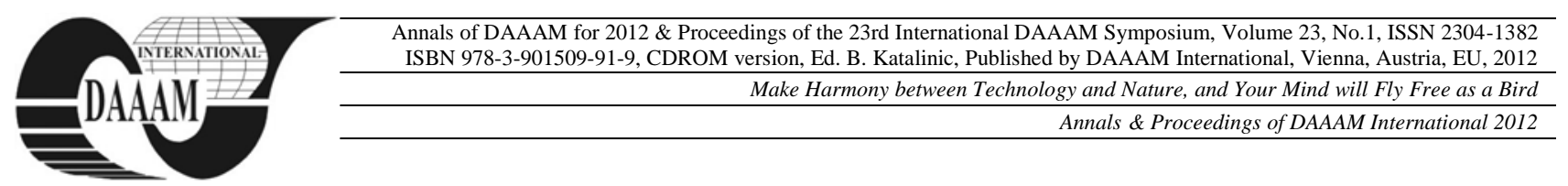

\title{
AUGMENTED REALITY APPLICATIONS IN MANUFACTURING ENGINEERING
}

\author{
NOVAK - MARCINCIN, J[ozef]; BARNA, J[ozef]; FECOVA, V[eronika] \& NOVAKOVA - \\ MARCINCINOVA, L[udmila]
}

\begin{abstract}
This article describes possibilities of manufacturing assembling process realized with using of special virtual elements of the augmented reality $(A R)$ and manufacturing processes with integration of parametrical CA data in virtual environment by graphical script. These possibilities are implemented in the virtual manufacturing environment of $A R$, where the application core allows engineers, designers and technologists not only to see important information about a position and orientation of the single manufactured product but also view on next item from technological process plan. By means of this the costumer can see the motion process of manufacturing process item according to its trajectory and necessary information about general characteristic of the manufacturing processes.
\end{abstract}

Keywords: augmented reality, virtual reality, manufacturing engineering, CA data, virtual tools

\section{INTRODUCTION}

This article brings the information on latest improvements in area of computer aided manufacturing engineering with utilization of elements of augmented reality (AR). Events and actions realized according this idea are defined as processes where real environment is put together with fractions of virtual one and this new conjunction presents to its user the augmented tool of a virtual working space. First part shortly describes new software application that is designated to support the manufacturing processes, improve their efficiency and to simplify training and educational activities in manufacturing area. Through the particular steps and working segments of this software application, there are main ideas and basic logical actions and principles of AR described. After general overview and description of current situation, the information is provided about new elements of application that help to improve its usability and present great progress from the viewpoint of working comfort together with assuring the propriety of realized product. Article outlines the science approach that is applied to the common activities of manufacturing process while using the tools presented in form of logical blocks which allow the data flow necessary for exploitation of AR [4, 8].

The first part of this article deals with basic programming element which concern application environment where the mentioned logical elements of AR were created. This introductory paragraph includes necessary information about augmented computer tools which were applied during the creation of this virtual application. By using these special virtual tools, the application allows constructer to manage an entire information data packet flow between different programming cores of the application. The next part of article provides the view on the individual logical zones where important data packets are harvested and compared with values from virtual arrays. After that, these data packets are sent to the displaying section of the manufacturing assembling application of the AR. In the next step, the chapter deals with solutions for production process of the AR where the logical loops allows for designer to see the process of the moving of the particular 3D model according the applying trajectory and the final position of all manufactured parts. In the end this article is focused on the special programming packet and improving elements which increase entire quality of the visual area of the application of $\mathrm{AR}[4,5]$.

\section{AUGMENTED REALITY UTILIZED IN MANUFACTURING ENGINEERING}

Supporting the manufacturing process with elements of augmented reality presents special kind of software solution and provides powerful hardware equipment for realization of products with large number of parts. Final product assembly includes all parts in right locations and with perfect functional characteristics while preventing it from assembly mistakes. The application of augmented reality for manufacturing defines the positions and orientations of handled subassemblies and their parts [6, 7].

There are a lot of issues that need to be solved during the computer aided design (CAD) of products in defined time. In the beginning the single $3 \mathrm{D}$ parts (individual entries of assembly list) needs to be created and described in all details. The 3D model comprises a pack of information about its properties (geometrical shape, orientation and position value, mass properties, material and structural characteristics). These data are usually sent to the special section of the calculation area of the computer aided engineering (CAE) systems. With use of these tools the models can be analyzed from different points of view and recommendations can be made concerning suitability of geometrical shape of the individual parts that are will be included in final assembly of the manufactured product. 3D CAD model filled with necessary information is then ready to be exported to system of augmented reality. In next stage the analyzed parts needs to be handled and provided with information about orientation and position, because it needs to be fixed in particular place of the basic production model in the real environment. Augmented reality application provides the designer with possibility to use different methods, while the characteristic feature means the technology of displaying and aiding of manufacturing positioning [2]. 


\section{APPLICATION OF AR FOR SUPPORTING OF MANUFACTURING PROCESSES}

Augmented reality system provides a complex view on handled area and relevant processes. Virtual parts are combined with real elements. It is a mutual existing of user's real scene together with computer's virtual scene what is considered as an augmentation. These methods of enhanced user environment find its utilization in many industrial spheres, for example in area production of parts from composite materials (Fig. 1).

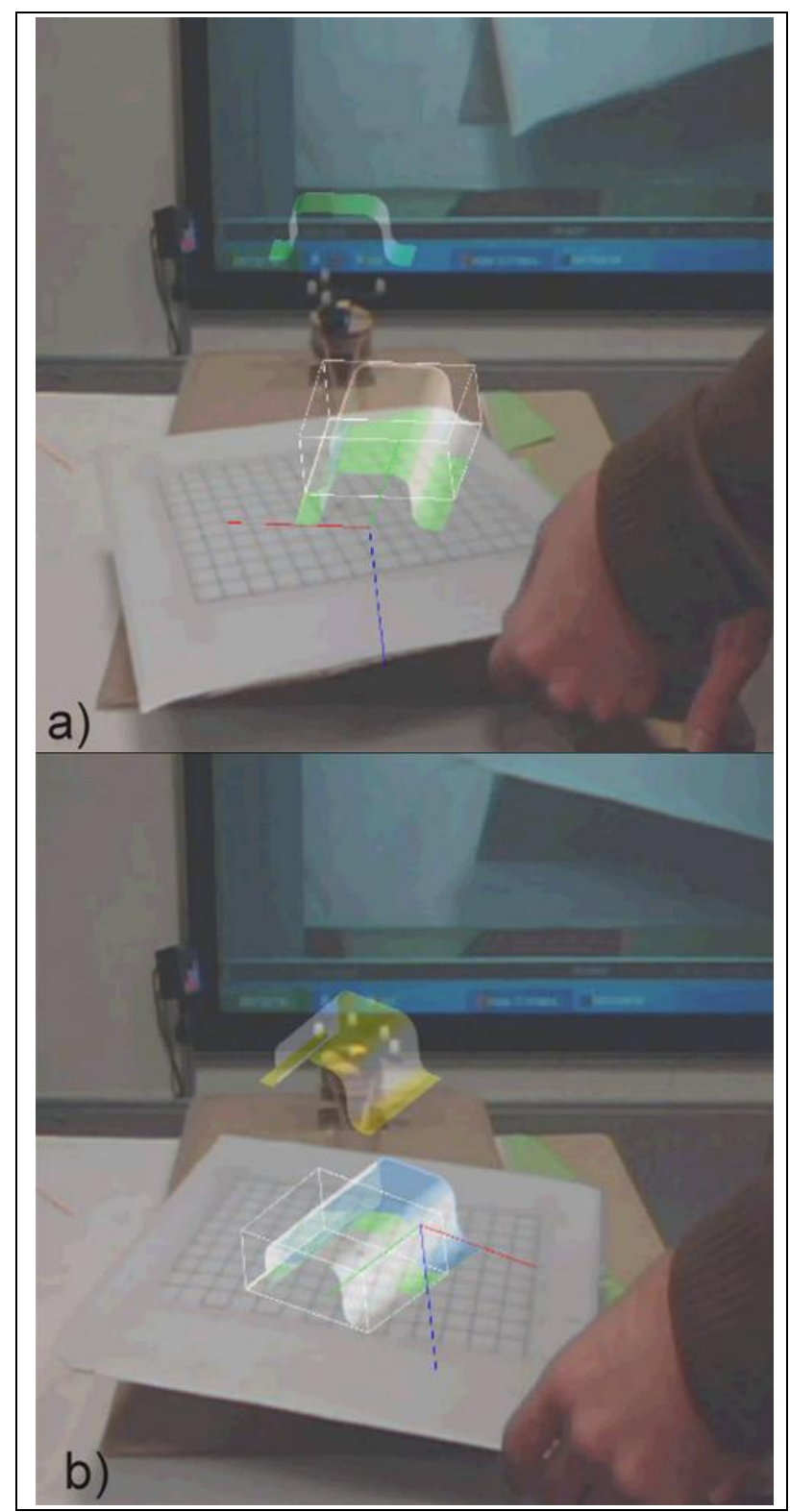

Fig. 1.Implementation of application for utilization of augmented reality in composite lay-up process (displaying of final state boundaries and individual layers applied)

Our team created application using the elements of augmented reality intended to be used as a supporting tool in manufacturing processes but can be used also in many other application areas. Following parts of this article describe the functional principles of this application. It is built on the mechanism that uses logical loops for realization of observation, collecting, evaluating and comparing activities and processing of all these obtained data in order to exploit them in final visualization output [5].

Programming environment called Virtools is based on principles of object programming, where different conditions, actions and relations are prescribed for particular objects that according to their function change to the so called building blocks of the application (BBs). Rules and actions running between individual blocks or their sections can be graphically expressed in form of behavior graph which at the same time serves as programming tool itself. Functionality of entire application can be then described through the tasks that are realized thanks to different behavior graphs.

Task of first behavior graph is to observe the position of the markers. Markers are graphical symbols situated in tracking space (working area). Their location and orientation is obtained using the special BBs called ART capture. Data are captured on the input pins of BBs and then the exact information about their positions get to the output area. On the basis of this application the user is able to collect, evaluate and to use the information about general movement in real working environment.

Presented behavior graph provides a view on general logical loops that are used for adjusting and comparison of information from ATR capture outputs (Fig. 2). BBs called Iterator can assign correct name of the part which should be applied into the virtual environment by order given by the logical loop. Thanks to that there are controlling instructions available: parameters of final vector, orientation and initial vector from previous step. All these data are concentrated in space called Loading section. Concurrently with this process all information about positions are sent and assigned to the BBs called Get Position which waits for activation before moving to the comparative section of the behavior graph. In the section for comparison, the received data are considered by various BBs to process and to evaluate those real data and the virtual ones. Coordinate system of the marker in the real environment is identified with the auxiliary system from virtual space. Such connection established with used of previously mentioned elements creates the platform for basic possibilities of augmented reality where real view is associated and overlapped with the 3D environment. New coordinate system obtains the important information from comparing section and logical loop procedure displays the process of moving the 3D part on its trajectory with parameters received from data array. To simplify, by means of the positional data from the marker of tracking system the application is able to assign the virtual coordinate system in the center of marker and use it for displaying purposes related to virtual part movement.

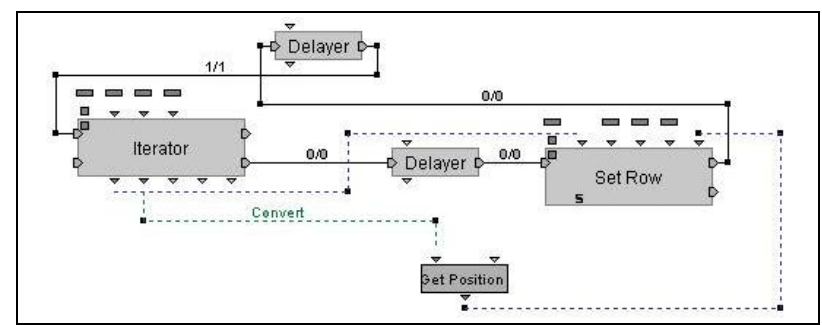

Fig. 2.General graphic scheme of behaviour graph created in Virtool programming environment 
Relevant data block of comparing section recalculates and evaluates necessary value of the $3 \mathrm{D}$ part to keep it identified with the real system. BBs that is working on Switch on Message principle constantly monitors the action of button reactivation. Displaying section then has two possibilities: to prefer moving trajectory of the 3D part or to provide value of the final vector position. After first button activation, the BBs Switch on Message receives the confirming message and necessary information about 3D model is sent from the data array to positional and comparing sections. Further confirmation inputs consequently continue to the displaying section where the final process of manufacturing of part is realized and the costumer is able to see the process of the moving of the 3D part according to its trajectory. This is repeated until the button is reactivated. Then the movement is stopped and substituted by the value of final position vector obtained from data array. If the button is pressed again, the BBs Iterator sends order to move to the next row in the data array. This way all new information of position and orientation are sent again into the logical sections of behavior graph with repeated testing, comparison and evaluation of new coming parameters. Displaying section shows the entire manufacturing process in part after part mode (Fig. 3) [5].

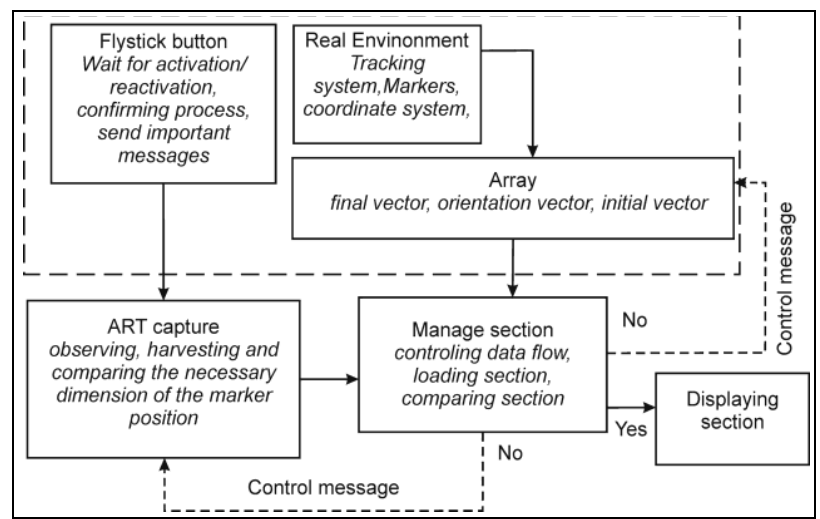

Fig. 3.Algorithm provides particular logical blocks where data from external devices are harvested and managed and then are sanded into the core of the general behaviour graph

\section{INTEGRATION OF PARAMETRICAL CA DATA TO AUGMENTED REALITY}

On Fig. 4 there are information provided about general steps of the process of displaying the important information such as data concerning the number of item in process plan, individual part name, material, total amount of relevant item in manufacturing in pieces, manufacturing mode (for example necessity of characteristic assembly procedure, type of joints, needed tools) and also the information about next upcoming assembly element. Beside these fact further information can be retrieved, such as distributor, mechanical and physical conditions, mass properties etc. This application comprises logical scripts for collection and translation of information from 3D CAD software. These data are then exported into the special array of the application. First they need to be completed in data packet, what means that all necessary information must to be filled using the special tools from CAD software. Information packet therefore includes necessary conditions and texts. It is compiled and saved into the section of computers hard drive at the typical informative formats like TXT or XLS.

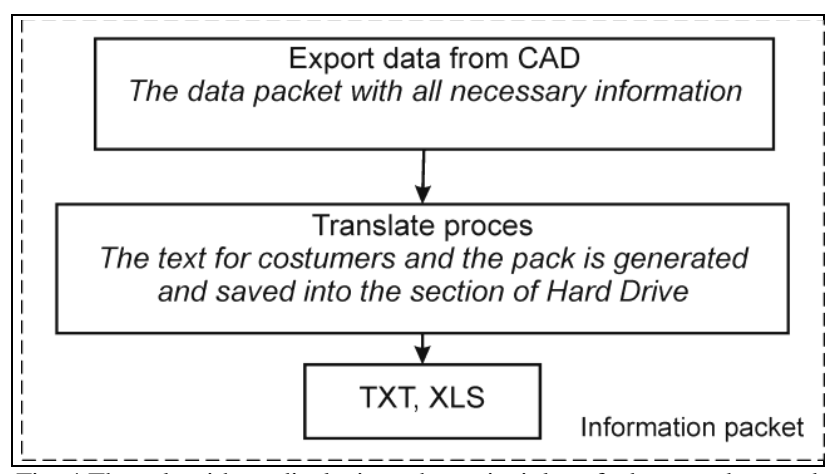

Fig. 4.The algorithm displaying the principle of data packet and translation to typical informative formats that are ready to be used for purposes of AR application

The packet is sent and translated into new behavior graph of the application. This operation of translation process is realized via special logical script which transforms and rebuilds this data packet to the individual entries (situated in rows and columns) of data array (Fig. 5). The logical script situated in behavior graph is used for reloading, classifying and assigning process of packet transformation in order to create the array form. After the transformation process is implemented into the data loop the new array implies exact information where needed. Described principles of logical core allow user to display relevant value from the data packet at any time. All the parameters that are needed in process of $3 \mathrm{D}$ movement according its trajectory are obtained from data array. Displayed moving part carries an exact value about final vector that is situated in the coordinate system. During this process, application enables the possibilities to send requirements and calls for operation. The logical script allows the enhancement of the working screen with chosen data, displaying them in real time without interrupting of visualization. Necessary information is prepared and shifted on the screen after confirming the particular button on the keyboard (A-activation of data package, S-Page Down, D-Page Up) [1].

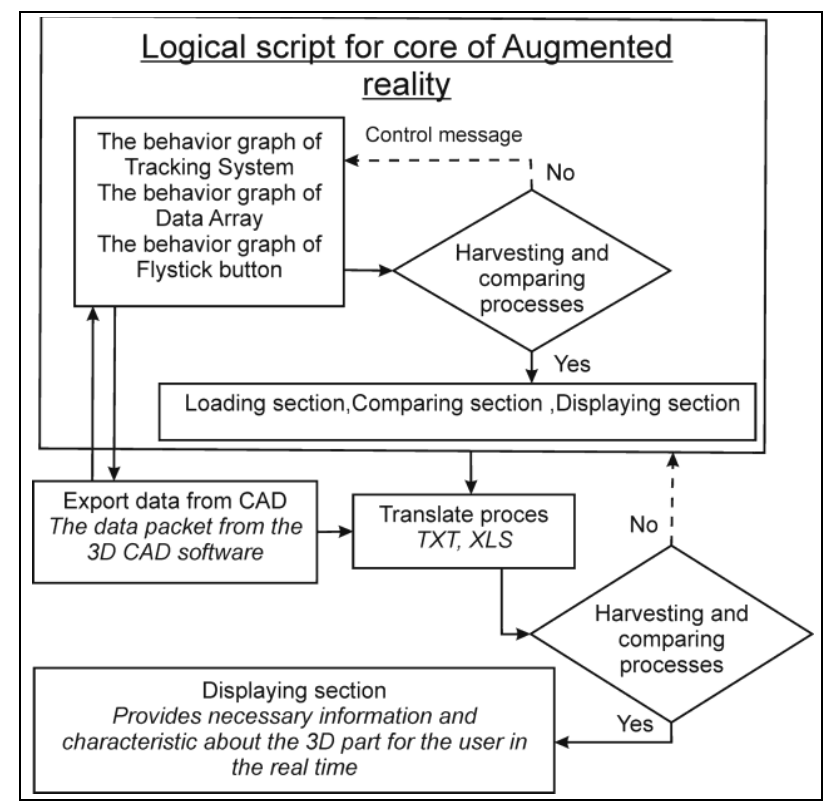

Fig. 5.The algorithm with logical scripts, blocks and sections for realization of application with AR 
When the logical core gets the information about changes in component numbering in the frame of data array (position information and details on movement trajectory of the 3D model in the data array of which position has been changed), the particular scripting form of the behavior graph reloads the new data packet (the previous packet is substituted by values from the new one) created for next model. By means of these processes, the application is ready to continually provide necessary information and characteristic about 3D parts for the user in the real time (Fig. 6).

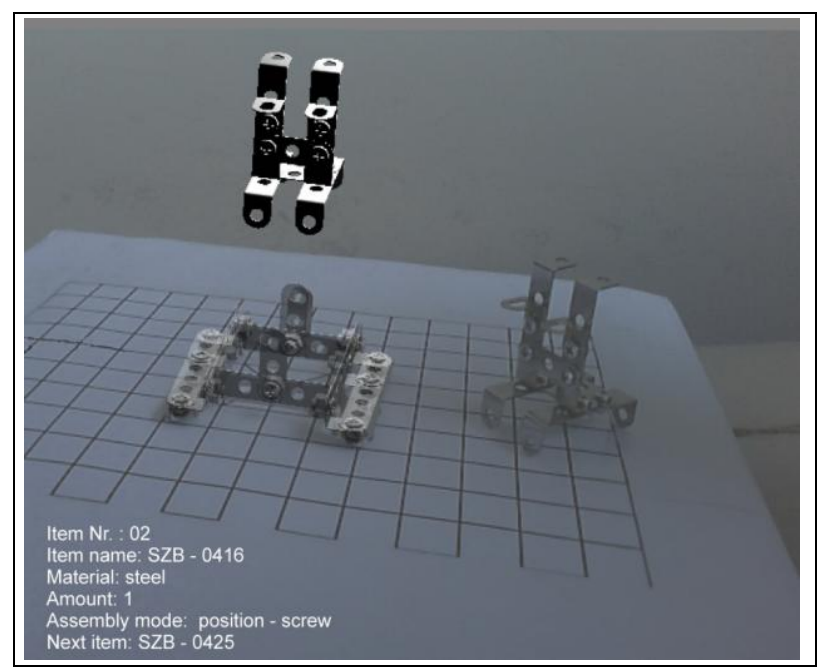

Fig. 6.Working screen providing selected information after confirmation of particular button on the keyboard

The example in next pictures provides the information about general steps contained in the process where user can see 3D form of information about that are to be applied in the working environment. The new logical loop imports data in the application. This additional information obtained on the user screen can present very strong support tool for any stage of manufacturing process (Fig. 7).

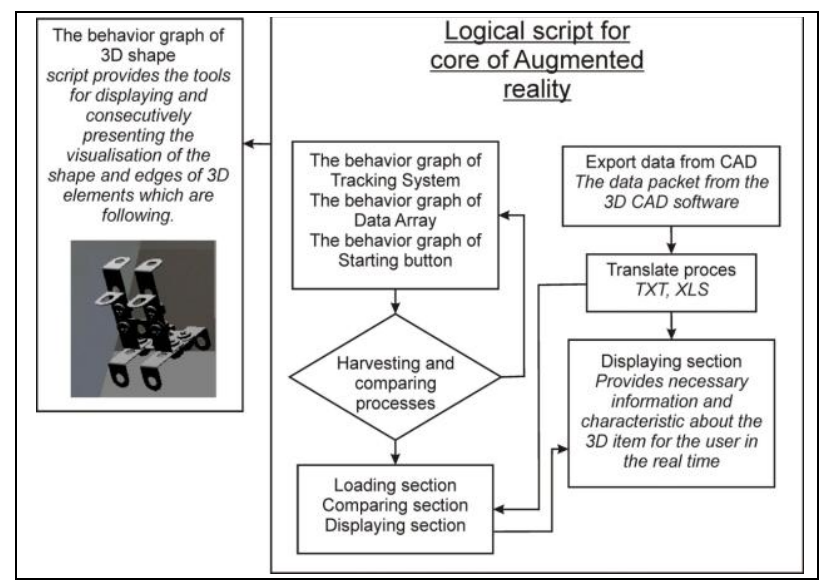

Fig. 7.The algorithm containing logical scripts that provide the 3D data like a geometry and shape of model

\section{CONCLUSION}

Elements of augmented reality in form of different virtual tools find their utilization in many industrial areas. Last decade predicted expanding of methods and applications that are based on principle of connection of real and virtual environments. On the example of real manufacturing process this article describes the principles and main theoretical fragments of augmented reality that can be implemented in real praxis. It focuses to the software application created in environment for objective programming. Main goal is to introduce the improvements and enhancements that were done and to point out the possibilities of their use together with benefits achieved for problem-free realization of manufacturing process in its entirety [3].

It gives new information about research goals marked out in the scientific area of application of $A R$ in manufacturing processes, what can provide not only powerfully tools for known tasks but also new possibilities in procedures exploitable in manufacturing and other industrial fields. Integration of parametrical CA data obtained from CAD models in the environment of augmented reality surely present the improvement of working comfort and quality of final results of realized tasks and this will next research area in future.

\section{ACKNOWLEDGEMENTS}

Ministry of Education, Science, Research and Sport of SR supported this work, contract VEGA No. 1/0032/12, KEGA No. 002TUKE-4/2012 and ITMS project 26220220125 .

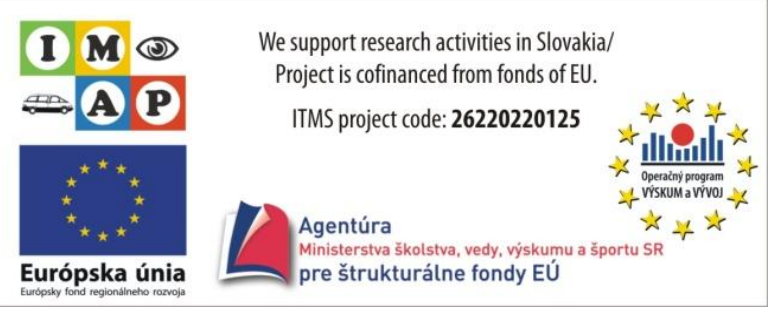

\section{REFERENCES}

[1] Gaspar, S.; Mascenik J. \& Pasko J. (2012). The effect of degassing pressure casting molds on the quality of pressure casting. Advanced Materials Research, No. 428, pp. 43-46, ISSN 1022-6680

[2] Kovacevic, D.; Budak, I.; Antic, A. \& Kosec, B. (2011). Special Finite Elements: Theoretical Background and Application. Tehnicki Vjesnik, Vol. 18, No. 4, pp. 649-655, ISSN 1330-3651

[3] Kuric, I. \& Kuba, J. (2006). Dynamic classification in CAPP Annals of DAAAM for 2006 \& Proceedings of the 17th International DAAAM Symposium, Vienna, Katalinic, B. (Ed.), pp. 215-216, DAAAM, Vienna

[4] Marcincin, J. N.; Brazda, P.; Janak, M. \& Kocisko, M. (2011) Application of virtual reality technology in simulation of automated workplaces. Tehnicki Vjesnik, Vol. 18, No. 4, pp. $577-$ 580, ISSN 1330-3651

[5] Marcincin, J. N.; Barna, J.; Janak, M.; Marcincinova, L. N. \& Fecova, V. (2011). Utilization of Open Source tools in assembling process with application of elements of augmented reality. Proceedings of VRCAI 2011: ACM SIGGRAPH Conference on Virtual-Reality Continuum and its Applications to Industry, pp. 427-430, ISBN 978-1-4503-1060-4, Hong Kong

[6] Matija, R.; Vojtko, I. \& Kocisko, M. (2011). Theoretical aspects of machining. In: Proceedings of the conference Transfer 2011, Casta Papiernicka. - Trencin, pp. 1-9, ISBN 978-80-8075-505-8

[7] Michalik, P.; Zajac, J.; Duplak, J. \& Pivovarnik, A. (2012). CAM software products for creation of programs for CNC machining. Lecture Notes in Electrical Engineering, Vol. 1, No. 141, pp. 421425, ISSN 1876-1100

[8] Ong S. K. \& Nee A. Y. C. (2004). Virtual and Augmented Reality Applications in Manufacturing, Springer-Verlag, London, ISBN $1-85233-796-6$ 